\title{
Preface to the Special Issue for Multiscale Multiphase Process Engineering (MMPE) 2011
}

Intrinsic to the production and design of equipment in the field of Chemical Process Engineering is the understanding of multiphase flows. Multiphase flow phenomena such as transport phenomena at material interfaces and the dynamics of material interfaces can be studied either through novel experimental techniques or novel computational fluid dynamics (CFD) algorithms. State-of-the-art multi-scale, hierarchical approaches for understanding multi-phase flow in Chemical Process Engineering have been developed which enable researchers to better understand the interaction of individual scales in multiphase flows.

The 1st International Symposium on Multiscale Multiphase Process Engineering (MMPE) was held in Kanazawa, Japan on 4-7 October, 2011 in order to discuss modern problems related to multiphase process engineering. An important purpose of the MMPE conference was to foster international collaborations amongst the participants. We were delighted to see that over 115 participants from many countries attended the MMPE symposium.

After the MMPE symposium, a call for papers to be submitted to a special issue of JCEJ, with the MMPE theme, was made to both conference participants and the readers of JCEJ. Thirty-three papers were submitted to this issue for review, and twentyeight papers were accepted through an iterative peer-review process. We hope that this special issue will provide a great opportunity to discuss issues related to multiscale multiphase process engineering and will be of help in disseminating to researchers the latest knowledge of multiscale multiphase process engineering.

We thank the authors for their contributions, and the reviewers in providing their thoughtful and constructive comments. We also express our gratitude to Prof. Hiroyuki Honda of Nagoya University, the Editor-in-Chief of JCEJ, for his substantial support. We thank Ms. Kazuko Yamashita, in the editorial office, for her assistance and devotion in the preparation of this special issue.

Chief Editor, Special Issue

Mitsuhiro Ohta, The University of Tokushima

Editors, Special Issue

Shunji Homma, Saitama University Shuichi Iwata, Nagoya Institute of Technology Tsutomu Nakazato, Kagoshima University

Koichi Terasaka, Keio University Makoto Yoshimoto, Yamaguchi University 